\title{
Enhanced effect of nuclear localization signal peptide during ultrasound-targeted microbubble destruction-mediated gene transfection
}

\author{
SHENG CAO*, QING ZHOU*, JIN-LING CHEN, NAN JIANG, \\ YI-JIA WANG, QING DENG, BO HU and RUI-QIANG GUO \\ Department of Ultrasound Imaging, Renmin Hospital of Wuhan University, Wuhan, Hubei 430060, P.R. China
}

Received February 4, 2016; Accepted March 3, 2017

DOI: $10.3892 / \mathrm{mmr} .2017 .6661$

\begin{abstract}
Ultrasound-targeted microbubble destruction (UTMD) can promote the entry of plasmid DNA (pDNA) into the cell cytoplasm, by increasing the permeability of the cell membrane. But the transfection efficiency remains low due to inability of the pDNA to enter the nucleus. Various methods have been explored to improve the UTMD transfection efficiency, but with little success. In cells, the classic nuclear localization signal (cNLS) peptide is an amino acid sequence that signals proteins that are due for nuclear transport. The present study aimed to investigate whether binding of a cNLS peptide to the pDNA may improve the transfection efficiency of UTMD. Four experimental groups were analyzed: Control group (UTMD + pDNA), group with cNLS (UTMD + pDNA + cNLS), group with mutated NLS (mNLS; UTMD + pDNA + mNLS), and group with cNLS and the nuclear import blocker, wheat germ agglutinin (WGA; UTMD + pDNA + cNLS + WGA). The NLS was labeled by fluorescein isothiocyanate, whereas pDNA was labeled with Cy3. Different molar ratios were tested for the NLS and pDNA combination in order to achieve optimal binding of the two molecules. Human umbilical vein endothelial cells were then transfected using the optimum ultrasonic irradiation parameters and NLS/pDNA molar ratio. At $6 \mathrm{~h}$ post-transfection, the rates of Cy3-labeled pDNA inside the cells and their nuclei were detected by flow cytometry and laser confocal microscopy, and the cellular vs. nuclear uptake of pDNA was calculated. In order to further evaluate the effect of NLS on UTMD-mediated gene transfection, the transfection efficiency and relative expression levels of mRNA
\end{abstract}

Correspondence to: Dr Rui-Qiang Guo, Department of Ultrasound Imaging, Renmin Hospital of Wuhan University, 238 Jiefang Road, Wuhan, Hubei 430060, P.R. China

E-mail: caosheng209@126.com

*Contributed equally

Key words: sonication, microbubbles, transfection, nuclear localization signal, ultrasound-targeted microbubble destruction and protein were detected at $48 \mathrm{~h}$ post-transfection. The results demonstrated that the optimal molar ratio of NLS with pDNA was $10^{4}: 1$. The rates of pDNA successful entry into the cell and nucleus were significantly higher in the cNLS group compared with the control group. The transfection efficiency, and relative expression levels of mRNA and protein from the plasmid were significantly increased in the cNLS group compared with the control group. The mNLS group displayed no significant difference compared with the control group, while the WGA group exhibited significant inhibition in most indicators of transfection efficiency compared to the cNLS group. These results suggest that combining a cNLS peptide with pDNA during UTMD-mediated transfection significantly improved transfection efficiency. Thus, a cNLS peptide may be an important mediator and a new strategy in enhancing the efficiency of UTMD-mediated gene transfection.

\section{Introduction}

Ultrasound-targeted microbubble destruction (UTMD) technology is as a novel method of non-viral vector gene transfection, that is increasingly favored by researchers. UTMD technology uses ultrasound microbubbles as nuclei to exert a cavitation effect, which increases the permeability of cell membranes during ultrasound exposure. The exogenous DNA can then enter and transfect a cell through the cell membrane (1). New methods are constantly explored in biomedical research in order to improve targeted gene transfection efficiency at levels comparable to viral vectors (1-3); these methods include the biotin-avidin system (4), the antigen-antibody targeting system $(5,6)$, and the novel ultrasound microbubbles system $(7,8)$. At present, these methods have limited success penetrating the barrier of cell membranes. In addition, for successful gene delivery, the nuclear membrane is a second barrier in eukaryotic cells as all materials must go through nuclear pore complexes to enter the nucleus. Micromolecules with molecular weight $<60 \mathrm{kDa}$ and diameter $<9 \mathrm{~nm}$ pass through nuclear pore complexes into the nucleus by passive diffusion, but macromolecule materials, such as plasmid DNA (pDNA), must contain nuclear localization signal (NLS) peptides, which mediate the active transport into the nucleus $(9,10)$. 
NLS peptides are composed of functional amino acid sequences and they are frequently used in the field of gene transfer research, as a method to enhance nuclear entry (11). Classic NLS (cNLS) peptides are rich in basic amino acids, such as arginine and lysine, and are a necessary signal for exogenous DNA or protein to enter into the nucleus. The nuclear transfer functions of the various NLS peptides are closely associated with their structure and sequence. The correct NLS sequence and structure is recognized by nuclear import proteins and is transported through the nuclear pore complex into the nucleus in the form of a cargo-import protein complex. cNLSs should be at the C-terminus of the cargo protein; if the position of the NLS is wrong, or if the sequence is reversed, the sequence is not recognized by the nuclear import protein, and thus the NLS loses its function (12). Furthermore, when a basic amino acid mutation is introduced in the cNLS sequence (e.g. ${ }^{126} \mathrm{PKKKRKV}{ }^{132}$ ), so that the lysine $(\mathrm{K})$ at position 128 is mutated to threonine (T), the resulting mutated NLS (mNLS) peptide loses its nucleus transport function $(13,14)$. Finally, exogenous lectin wheat germ agglutinin (WGA) has been reported to act as a nuclear blocker, sealing the nuclear pore and preventing the NLS peptides from interacting with the nuclear transport receptor $(15,16)$. Thus, in the presence of WGA, a targeted protein or gene is not able to enter into the nucleus, even if it contains a functional cNLS $(15,16)$.

The present study aimed to investigate novel methods to improve UMTD-mediated gene transfection. Based on the property of NLS peptides to enhance nuclear entry, the hypothesis that cNLS may act as a mediator to increase pDNA entering the nucleus during UMTD was examined. The present findings suggested that cNLS may be a potential strategy to improve the transfection efficiency of the novel UTMD gene transfection technology.

\section{Materials and methods}

Plasmid labeling. The plasmid hANGPT1-EGFP (cat. no. GOSE63322; element sequence, CMV-MCSEGFP-SV40-Neomycin) was constructed by GeneChem Technology Co., Ltd. (Shanghai, China), amplified in Escherichia coli, and then purified with the Wizard ${ }^{\mathrm{TM}}$ Maxiprep DNA Purification System (Promega Corporation, Madison, WI, USA). In hANGPT1-EGFP, the angiopoietin 1 (ANGPT1) coding region was cloned in the vector GV230, under the control of the human cytomegalovirus promoter/enhancer and followed by the enhanced green fluorescent protein (EGFP) protein, for visualization of translation efficiency (Fig. 1). In addition, pDNA was labeled with Cy3, using a Label IT Tracker Intracellular Nucleic Acid Localization kit (Mirus Bio, LLC, Madison, WI, USA), according to the manufacturer's protocol. The reagent to plasmid weight ratio was 1:2, and the labeled Cy3-pDNA was purified by ethanol precipitation and resuspended to a concentration of $1 \mu \mathrm{g} / \mu \mathrm{l}$.

Combination of NLS and plasmid hANGPT1-EGFP. The SV40 T antigen NLS peptide (PKKKRKV; molecular weight, 883 Da; cat. no. PO15071402) and mutated NLS peptide (PKTKRKV; molecular weight, 856 Da; cat. no. PO16022402) were synthesized and high-performance liquid chromatography (HPLC)-purified by Multiple Peptide Systems (San
Diego, CA, USA). NLS peptides were non-covalently bound to supercoiled phANGPT1-EGFP plasmid by incubation in $4 \mathrm{X}$ phosphate buffer saline (PBS; $547 \mathrm{mM} \mathrm{NaCl}, 10.7 \mathrm{mM}$ $\mathrm{KCl}, 40.6 \mathrm{mM} \mathrm{Na} \mathrm{HPO}_{4}, 7.1 \mathrm{mM} \mathrm{KH} \mathrm{PO}_{4}, \mathrm{pH}$ 7.1) for $30 \mathrm{~min}$ at room temperature. Different molar ratios were tested as follows: $0: 1,5 \times 10^{2}: 1,10^{3}: 1,5 \times 10^{3}: 1,10^{4}: 1$, and $5 \times 10^{4}: 1$ (NLS:phANGPT1-EGFP) The efficiency of binding was tested by agarose gel electrophoresis (Geliance 200; Perkin Elmer, Inc., Waltham, MA, USA), and the lowest molar ratio that resulted in a bound complex was determined as the optimal binding condition.

The NLS peptide was labeled with fluorescein isothiocyanate (FITC; Wuhan Boster Biological Technology, Ltd., Wuhan, China). A total of $1 \mathrm{M} \mathrm{NLS,} 40 \mathrm{mM}$ FITC and $2 \mathrm{M}$ DIEA (Suihou Hongqi Chemical Co., Ltd., Suizhou, Hubei, China) were mixed in the reactor and then placed on a shaking bed at $30^{\circ} \mathrm{C}$ for $2 \mathrm{~h}$. pDNA was labeled with $\mathrm{Cy} 3$ as above. The bound complex was observed by overlapping the two types of fluorescence under an inverted fluorescence microscope (IX51; Olympus Corporation, Tokyo, Japan).

Cell culture. Human umbilical vein endothelial cells (HUVECs) were supplied by the medical center of Renmin Hospital, Wuhan University (Wuhan, China). HUVECs were cultured in endothelial cell medium (ECM; ScienCell Research Laboratories, Inc., Carlsbad, CA, USA). The cells were maintained in $10 \mathrm{~cm}$ culture dishes at $37^{\circ} \mathrm{C}$ in a humidified and $5 \% \mathrm{CO}_{2}$ atmosphere. For transfection experiments, cells were trypsinized and seeded at a density of $1 \times 10^{6}$ cells/well in two 6-well plates $24 \mathrm{~h}$ prior to transfection. One of the 6 -well plates was transfected using UTMD with FITC-NLS and Cy3-labeled pDNA, to observe the subcellular localization following $6 \mathrm{~h}$ of transfection, using a fluorescent microscope and laser confocal microscope (FV1200; Olympus Corporation), respectively. The other 6-well plate was transfected with NLS and pDNA, for evaluation of transfection efficiency and cell survival.

Microbubble preparation. The contrast agent Sulphur hexafluoride microbubbles, named SonoVue (Bracco Imaging SPA, Milan, Italy), was prepared as per the manufacturer's instructions. Lyophilized powder was reconstituted with $5 \mathrm{ml} 0.9 \%$ sodium chloride w/v solution following vigorous oscillation, then a suspension containing $\sim 2 \times 10^{8}$ microbubbles $/ \mathrm{ml}$ was obtained. The microbubbles were filled with sulfur hexafluoride gas and encapsulated by a thin and flexible monolayer of phospholipids. The diameters of the majority of microbubbles were $2-5 \mu \mathrm{m}$.

UTMD-mediated plasmid transfection. The samples in the present study were divided into 4 groups as follows: Control group, UTMD + pDNA; cNLS group, UTMD + pDNA + cNLS; mutated NLS (mNLS) group, UTMD + pDNA + mNLS; nuclear import blocker WGA group, UTMD + pDNA +cNLS + WGA. Gene transfection was performed by UTMD when the cells in the 6-well plates were $70-90 \%$ confluent. The culture medium was replaced with 4 ml OptiMEM ${ }^{\circledR}$ medium (Gibco; Thermo Fisher Scientific, Inc., Waltham, MA, USA) containing $400 \mu \mathrm{l}$ microbubbles per well and $60 \mu \mathrm{g}$ pDNA, with or without NLS. Subsequently, ultrasound irradiation was performed on the cells and the plasmid-microbubble suspension, using the 
UGT2007 ultrasound irradiation system (Ultrasonic Research Institute of Chongqing Medical University, Chongqing, China). The parameters of ultrasonic irradiation were selected based on optimization experiments reported in a previous study from our group (17), as follows: Probe was placed upper the cells, and irradiation was performed with intensity of $1.5 \mathrm{~W} / \mathrm{cm}^{2}$, frequency of $1 \mathrm{MHz}$, irradiation time of $30 \mathrm{sec}$, duty cycle of $30 \%$, microbubble concentration of $1 \times 10^{7} / \mathrm{ml}$ and plasmid concentration of $15 \mu \mathrm{g} / \mathrm{ml}$. Each experiment was repeated three times. The cell culture medium was replaced with ECM medium at $24 \mathrm{~h}$ post-transfection.

Cellular and nuclear uptake of pDNA. HUVECs were transfected with Cy3-labeled pDNA and then incubated at $37^{\circ} \mathrm{C}$ and $5 \% \mathrm{CO}_{2}$ atmosphere for $6 \mathrm{~h}$. HUVECs were then washed with PBS and harvested with trypsin (Beyotime Institute of Biotechnology, Nantong, China). The number of fluorescent cells was detected by flow cytometry, and the percent of Cy3-positive cells over total represented the cellular uptake efficiency of pDNA.

In order to calculate the nuclear uptake of pDNA, the cells were seeded on sterile coverslips placed on the bottom of a 6-well plate and transfected as described above. Cells were then fixed with $4 \%$ paraformaldehyde (Beyotime Institute of Biotechnology) for $10 \mathrm{~min}$ at room temperature. The membrane was permeabilized by $0.2 \%$ Triton X-100 diluted in PBS for 8-10 min at room temperature. Finally, DAPI was added to the cells in the dark as a nuclear counterstain, and the cells were washed with PBS three times for 3-5 min prior to observation. The subcellular localization of the Cy3-labeled pDNA was observed under a laser confocal microscope. The fluorescence intensity (FL) of the whole cell $\left(\mathrm{FL}_{\mathrm{cell}}\right)$ and the nucleus ( $\mathrm{FL}_{\text {nucleus }}$ ) was quantified using Image $\mathrm{J}$, version 1.48 software (http://rsb.info.nih.gov/ij; National Institutes of Health, Bethesda, MD, USA). The percentage of $\mathrm{FL}_{\text {nucleus }} / \mathrm{FL}_{\text {cell }}$ represented the nuclear uptake efficiency of the pDNA.

Cell viability. Cell viability was measured using the Cell Counting Kit-8 (CCK-8) assay (Dojindo Molecular Technologies, Inc., Kumamoto, Japan), as per the manufacturer's instructions. Briefly, at $48 \mathrm{~h}$ post-transfection, HUVECs were seeded in a 96-well plate at $\sim 5 \times 10^{3}$ cells/well in $90 \mu$ l culture medium. Untransfected HUVECs were used as a control, and equal volume of ECM medium without any cells was used as a blank measurement. Following incubation at $37^{\circ} \mathrm{C}$ and $5 \%$ $\mathrm{CO}_{2}$ atmosphere for $24 \mathrm{~h}, 10 \mu \mathrm{l}$ CCK- 8 was added to each well for $4 \mathrm{~h}$, and then absorbance was measured at $450 \mathrm{~nm}$ using an automatic microplate reader (Lambda 1050; Perkin Elmer, Inc., Houston, Texas, USA). Six duplicate experiments were performed for each sample. Cell viability was calculated using the optical density $(\mathrm{OD})$ as follows: cell viability $(\%)=\left(\mathrm{OD}_{\text {sample }}\right.$ $\left.-\mathrm{OD}_{\text {blank }} / \mathrm{OD}_{\text {control }}-\mathrm{OD}_{\text {blank }}\right) \times 100$.

Transfection efficiency. At $48 \mathrm{~h}$ post-transfection, up to $500 \mu \mathrm{l}$ of cell suspension from each group was analyzed by flow cytometry (FACSCalibur; BD Biosciences, Franklin Lakes, NJ, USA). CELLQuest software version 3.0 was used for analysis (BD Biosciences). HUVEC transfection efficiency was calculated as follows: Transfection efficiency=the ratio of fluorescent-positive cells per total $\mathrm{x}$ cell viability.
Reverse transcription-polymerase chain reaction ( $R T-P C R)$. At $48 \mathrm{~h}$ post-transfection, total RNA was extracted using the TRIzol reagent (Aidlab Biotechnologies Co., Ltd, Beijing, China), cDNA was obtained by reverse transcription and the mRNA expression of the hANGPT1 gene was semi-quantitatively analyzed using the Applied Biosystems 7500FAST RT-PCR system and SYBR Green (Thermo Fisher Scientific, Inc.). The primers were as follows: hANGPT1, forward 5'-CGT GGAACCGGATTTCTCTT-3' and reverse 5'-GTACTGCCT CTGACTGGTAATG-3'; and GAPDH, forward 5'-CAAGGT CATCCATGACAACTTTG-3' and reverse 5'-GTCCACCAC CCTGTTGCTGTAG-3'. The reaction conditions were $50^{\circ} \mathrm{C}$ for $2 \mathrm{~min}, 95^{\circ} \mathrm{C}$ for $10 \mathrm{~min}$ and then 40 cycles of $95^{\circ} \mathrm{C}$ for $30 \mathrm{sec}$ and $60^{\circ} \mathrm{C}$ for $30 \mathrm{sec}$. PCR products were electrophoresed on a $1 \%$ agarose gel and stained with ethidium bromide. GAPDH was used to normalize cDNA from different samples. The relative expression of hANGPT1 to GAPDH was semi-quantified by a gel imaging analysis system (Geliance 200; Perkin Elmer, Inc.).

Western blot. At $48 \mathrm{~h}$ post-transfection, analysis of the hANGPT1 protein expression was performed by western blotting. Cells (1x10\%/sample) were collected and radioimmunoprecipitation (RIPA) buffer (Beyotime Institute of Biotechnology) was used as the lysis buffer. A total of $200 \mu 1$ RIPA was added per well for 3-5 min at room temperature and then placed on ice for $30 \mathrm{~min}$. Samples were repeatedly agitated to ensure full cell pyrolysis. Samples were then centrifuged at $4^{\circ} \mathrm{C}$ and $12,900 \mathrm{xg}$ for $5 \mathrm{~min}$. The supernatant was collected as the total protein solution. A bicinchoninic protein concentration determination kit (Beyotime Institute of Biotechnology) was used to quantify the concentration of protein according to the manufacturer's instructions. Total protein $(50 \mu \mathrm{g})$ was loaded into a $10 \%$ sodium dodecyl sulfate-polyacrylamide gel to separate the protein. The separated proteins were transferred to polyvinylidene fluoride membranes (EMD Millipore, Billerica, MA, USA) and blocked with 5\% non-fat dry milk (Aspen Bio, Inc., Wuhan, Hubei, China) for $1 \mathrm{~h}$ at room temperature. The membranes were washed with TBST (TBS with $0.1 \%$ Tween-20) then incubated with anti-hANGPT1 antibody (1:1,000; cat. no. ab133425; Abcam, Cambridge, MA, USA) at $4^{\circ} \mathrm{C}$ overnight. Following incubation with horseradish peroxidase-coupled secondary antibodies (1:10,000; cat. no. BA1054; Boster Biotechnology, Wuhan, China) for $1 \mathrm{~h}$ at room temperature. The membranes were washed in TBST buffer containing 0.1\% Tween-20 (Aspen Bio, Inc.) and protein expression was detected using a electrochemiluminescence kit (cat. no. NCI5079; Thermo Fisher Scientific, Inc.). The X-ray film was scanned and the AlphaEaseFC software (version 4.0.0; Alpha Innotech Corporation; ProteinSimple; Bio-Techne, Minneapolis, MN, USA) was used to analyze the optical density. The relative expression of hANGPT1 to GAPDH was measured.

Statistical analysis. Quantitative data are presented as mean \pm standard error, and statistical analysis was performed using SPSS 19.0 software package (IBM SPSS, Armonk, NY, USA). The parameters of the four groups were compared using a completely randomized design analysis of variance. Results with significant differences were further analyzed by post hoc 
tests to compare the difference between two groups. $\mathrm{P}<0.05$ was considered to indicate a statistically significant difference.

\section{Results}

Combination of NLS and plasmid hANGPT1-EGFP. At $6 \mathrm{~h}$ post-transfection, green fluorescence-labeled NLS and red fluorescence-labeled pDNA were detected at the same locations and with similar signal intensity within the cell, and this finding suggested a potential combination between them (Fig. 2). The molecular weight of a potential NLS and plasmid complex should be increased compared with the plasmid alone. In addition, the NLS is positively charged while DNA is negatively charged, therefore their interaction should partially neutralize electrostatic charge. Both these factors, i.e. neutralized electrostatic charge and increase in molecular weight, lead to slower band migration in gel electrophoresis, until complete blocking is achieved. Agarose gel electrophoresis analysis demonstrated that the optimum molar ratio of NLS/ plasmid combination was $10^{4}: 1$ (Fig. 3). When the molar ratio was $10^{4}: 1$ or greater, the NLS and plasmid complex was completely immobilized in the sample well (Fig. 3).

Distribution of FITC-NLS in cells. At $6 \mathrm{~h}$ post-transfection, the distribution of green fluorescence FITC-labeled NLS in the cells was observed in the cNLS, mNLS and WGA groups by fluorescence microscopy. Most green fluorescence signal was located in the nuclei in the cNLS group (Fig. 4). Green fluorescence signal was located in both the cytoplasm and the nuclei in the mNLS group, while it was almost exclusively in the cytoplasm in the WGA group (Fig. 4). These findings revealed that the cNLS peptide successfully entered into the nucleus following UTMD-mediated transfection, while mNLS only partly entered into the nucleus and WGA effectively inhibited cNLS nuclear entry.

cNLS promotes the cellular and nuclear uptake of pDNA. At $6 \mathrm{~h}$ post-transfection, the rates of pDNA uptake into the cells were $61 \pm 11,80 \pm 10,55 \pm 9$ and $58 \pm 10 \%$ for the control, cNLS, mNLS and WGA groups, respectively (Fig. 5A). The rates of pDNA uptake into the nucleus were $20 \pm 4,50 \pm 11,18 \pm 3$ and $10 \pm 3 \%$ for the control, cNLS, mNLS and WGA groups, respectively (Fig. 5B). These results indicated that the cNLS significantly enhanced the cellular and nuclear uptake of pDNA during UTMD-mediated gene transfection.

Effect of cNLS on cell viability and transfection efficiency. At 48 h post-transfection, the cell viability was $>80 \%$ in all 4 experimental groups, and no significant difference was observed among the groups $(\mathrm{P}>0.05 ;$ Fig. $6 \mathrm{~A})$. The transfection efficiency was $40 \pm 4,61 \pm 10,37 \pm 5$ and $23 \pm 4 \%$ in the control, cNLS, mNLS and WGA groups, respectively. The transfection efficiency in the cNLS group was significantly increased compared with the control ( $57 \%$ increase), mNLS and WGA groups $(\mathrm{P}<0.05$; Fig. 6B). This finding indicated that the cNLS promoted UTMD-mediated transfection efficiency of pDNA. Transfection efficiency was also determined by expression of EGFP, encoded on the pDNA vector, via flow cytometry and fluorescence microscopy. The number of cells expressing EGFP markedly increased in the cNLS group compared with

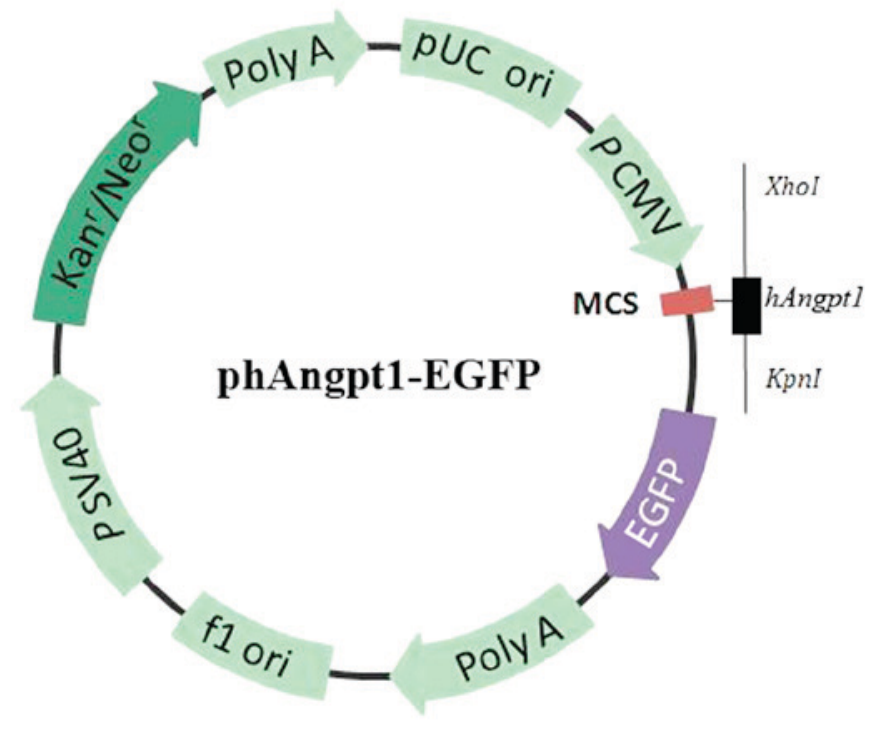

Figure 1. Structure of the plasmid hANGPT1-EGFP. The sequence encoding hANGPT1 is inserted downstream of the CMV promoter. The EGFP cDNA follows the hANGPT1. hANGPT1, human angiopoietin 1; EGFP, enhanced green fluorescence protein; CMV, cytomegalovirus; $\mathrm{Kan}^{\mathrm{r}}$, kanamycin; $\mathrm{Neo}^{\mathrm{r}}$, neomycin; poly A, poly adenosine; ori, origin of replication; MCS, multiple cloning site.

the other three groups, confirming enhanced transfection efficiency (Fig. 7).

cNLS enhances the expression of hANGPT1 $m R N A$ and protein. The relative expression levels of hANGPT1 mRNA were $28 \pm 4,55 \pm 8,27 \pm 4$ and $17 \pm 3 \%$ in the control, cNLS, mNLS and WGA groups, respectively, with the cNLS group displaying the highest mRNA expression levels compared with the other three groups $(\mathrm{P}<0.05$; Fig. $8 \mathrm{~A})$. The relative expression levels of the hANGPT1 protein were $22 \pm 4,46 \pm 8,22 \pm 5$ and $12 \pm 3 \%$ in the control, cNLS, mNLS and WGA groups, respectively, with the cNLS group again displaying the highest protein expression levels compared with the other three groups $(\mathrm{P}<0.05$; Fig. 8B). Expression of hANGPT1 mRNA and protein in the mNLS group was not significantly different to the control group, whereas the WGA group displayed decreased expression compared with the control group ( $\mathrm{P}<0.05$; Fig. 8).

\section{Discussion}

As a new type of non-viral vector gene transfection, UTMD technology uses ultrasound microbubbles as nuclei to exert a cavitation effect, which increases the permeability of cell membranes during ultrasound exposure (1,18-21). Exogenous DNA can enter a cell through the cell membrane and transfect the cell under the appropriate conditions. The barrier function of the cell and nuclear membranes, and nuclease degradation, are some of the limiting factors of transfection efficiency by UTMD-mediated exogenous gene in eukaryotic cells. Several methods have been tested in order to improve transfection efficiencies: The biotin-avidin system can gather large amounts of exogenous DNA around the microbubbles; the antigen-targeted antibody system can identify the damaged endothelial cells specifically; a new type of ultrasound microbubbles can change their physical and chemical properties; 

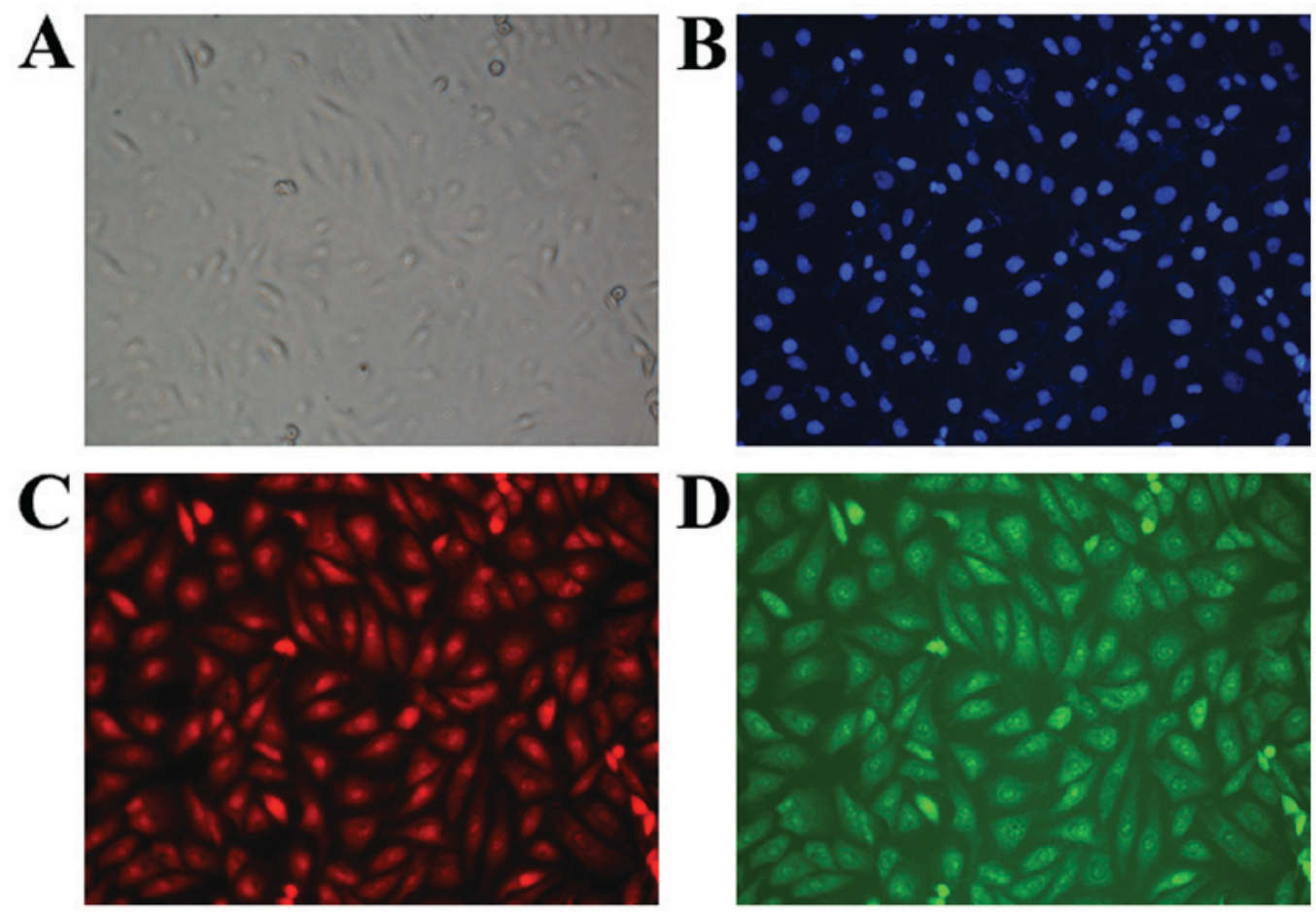

Figure 2. Human umbilical vein endothelial cells were transfected with plasmid DNA and NLS peptide by ultrasound-targeted microbubble destruction method (magnification, x400). (A) Cells imaged by bright-field microscopy. (B) DAPI-stained nuclei (blue). (C) Cy3-labeled plasmid DNA (red). (D) Fluorescein isothiocyanate-labeled NLS (green). NLS, nuclear localization signal.

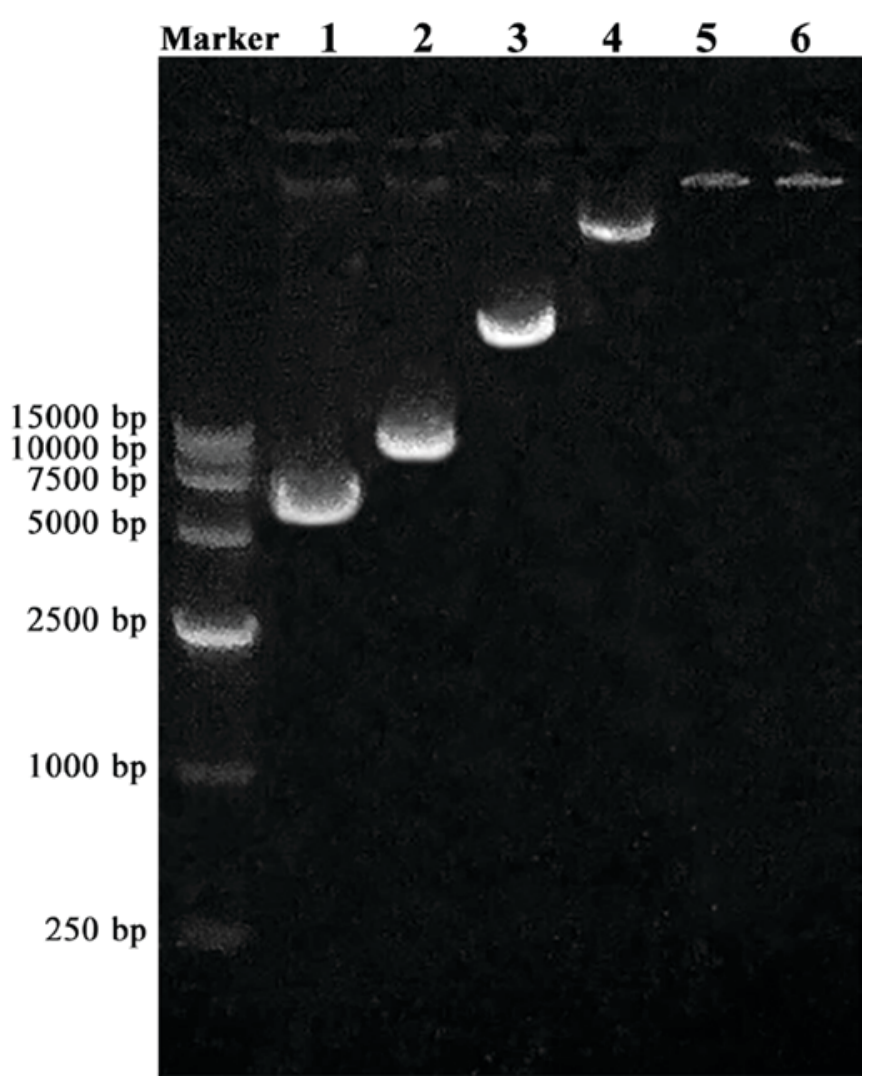

Figure 3. NLS-pDNA binding was evaluated by agarose gel electrophoresis at different NLS/pDNA molar ratios. Lane 1, 0:1; lane 2, 5x10²:1; lane 3, $10^{3}: 1$; lane $4,5 \times 10^{3}: 1$; lane $5,10^{4}: 1$; and lane $6,5 \times 10^{4}: 1$. When the molar ratio of NLS/pDNA combination was $10^{4}: 1$ (lane 5) or greater, the NLS-pDNA complex was immobilized in the sample well. The DNA molecular weight marker was $\lambda$ /Hind III. NLS, nuclear localization signal; pDNA, plasmid DNA. and addition of cationic polymers can improve the transfection efficiency $(22,23)$. These methods have some effect in penetrating the cell membrane barrier, but have provided little benefit to cross the nuclear membrane barrier. Macromolecules, such as pDNA, must contain an NLS in order to be actively transported into the nucleus, by specific interaction of the NLS with the nuclear import proteins $(9,10)$.

NLS peptides are positively charged because their sequence is rich in arginines and lysines, and therefore can bind negatively charged pDNA by electrostatic interactions (24). The present study confirmed that the NLS and pDNA combined in a complex and the optimum molar ratio for this binding was $10^{4}: 1 \mathrm{NLS} / \mathrm{pDNA}$, as detected by agarose gel electrophoresis, performed similarly to a previous study by Duvshani-Eshet et al (25). For different exogenous DNAs, the differences in molecular weight and electrostatic charge result in different molar ratios for binding. Part of the positively charged NLS can also interact with the negatively charged SonoVue microbubbles, thereby increasing pDNA uptake into cells indirectly during UTMD; this phenomenon may also partly explain the higher transfection efficiency in the cNLS group compared with the control group.

The nuclear import of NLS-mediated exogenous DNA is divided into at least two steps. The first step is the process of NLS identification by the NLS receptor, which then forms a nuclear pore positioning complex gathered on the cytoplasmic side of the nuclear membrane. The second step is the process of nuclear transfer, which is dependent on temperature and energy, and results in the NLS peptide passing through the nuclear pores and into the nucleus (26). When the ultrasound cavitation effect is generated by UTMD, the absorption or dissipation of the ultrasonic energy can cause a local and 


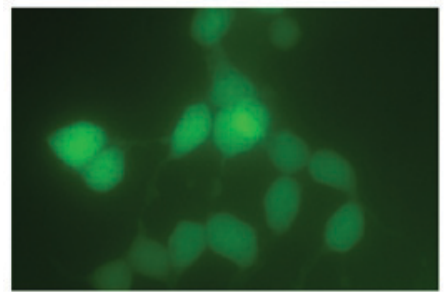

cNLS

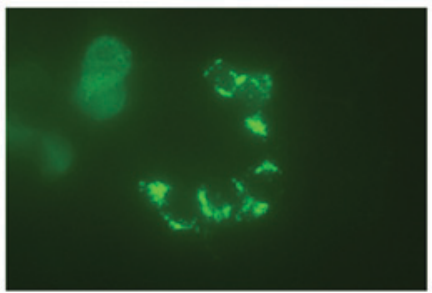

mNLS

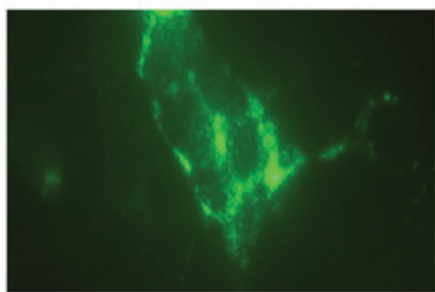

cNLS+WGA

Figure 4. Distribution of FITC-labeled NLS in transfected cells (magnification, $\mathrm{x} 1,000$ ). Almost all green fluorescence was observed in the nucleus in the cNLS group. Green fluorescence was observed in both the cytoplasm and the nucleus in the mNLS group, whereas green fluorescence mostly appeared in the cytoplasm and almost no nuclear fluorescence in the WGA group. NLS, nuclear localization signal; c, classic; m, mutated; WGA, wheat germ agglutinin.
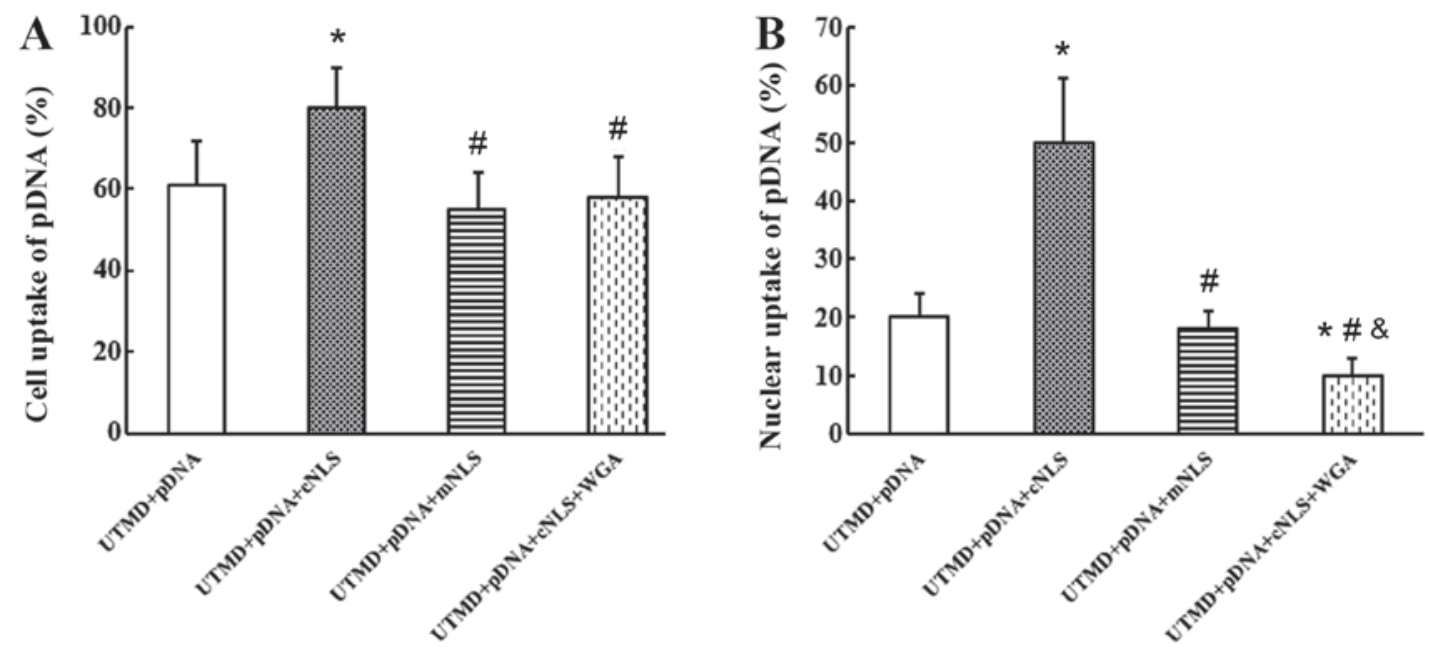

Figure 5. Rates of pDNA uptake in the (A) cell and (B) nucleus in human umbilical vein endothelial cells in the control group (UTMD + pDNA), cNLS group (UTMD + pDNA + cNLS), mNLS group (UTMD + pDNA + mNLS), and WGA (UTMD + pDNA + cNLS + WGA) group. " $\mathrm{P}<0.05$ vs. control; " $\mathrm{P}<0.05$ vs. cNLS; and ${ }^{\circledR} \mathrm{P}<0.05$ vs. mNLS. pDNA, plasmid DNA; UTMD, ultrasound-targeted microbubble destruction; NLS, nuclear localization signal; c, classic; m, mutated; WGA, wheat germ agglutinin.
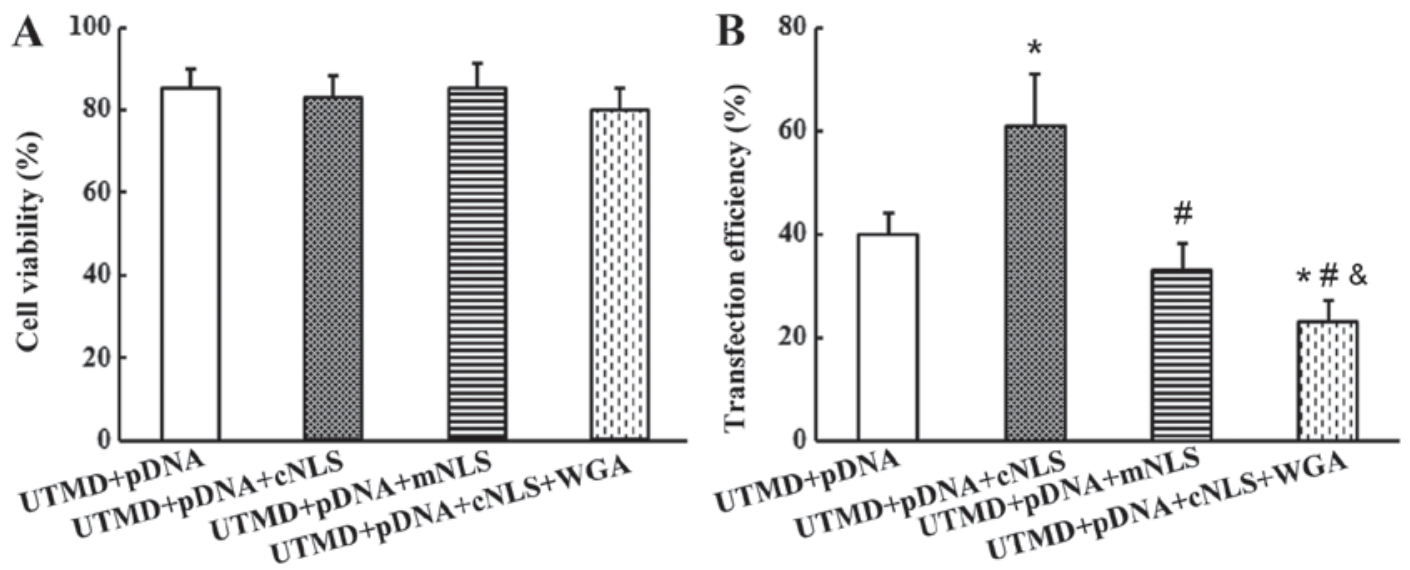

Figure 6. (A) Cell viability and (B) transfection efficiency in control group (UTMD + pDNA), cNLS group (UTMD + pDNA + cNLS), mNLS group (UTMD + pDNA + mNLS), and WGA (UTMD + pDNA + cNLS + WGA) group. " $\mathrm{P}<0.05$ vs. control; ${ }^{\prime} \mathrm{P}<0.05$ vs. cNLS; and ${ }^{\circledR} \mathrm{P}<0.05$ vs. mNLS. UTMD, ultrasound-targeted microbubble destruction; pDNA, plasmid DNA; NLS, nuclear localization signal; c, classic; m, mutated; WGA, wheat germ agglutinin.

transient temperature increase, which may be advantageous to the exogenous DNA to get into the cytoplasm and may provide energy to promote the NLS-mediated pDNA entry into the nucleus $(2-3,27)$. This phenomenon may also explain the NLS-mediated exogenous DNA entry into the nucleus observed in the present study; the rates of cell and nuclear
pDNA uptake increased significantly when the transfection was conducted by UTMD combined with cNLS.

In the present study, the rates of cell and nuclear uptake of pDNA both increased following addition of the optimal proportion of cNLS/pDNA. The transfection efficiency also improved significantly, as well as expression of mRNA and protein of 

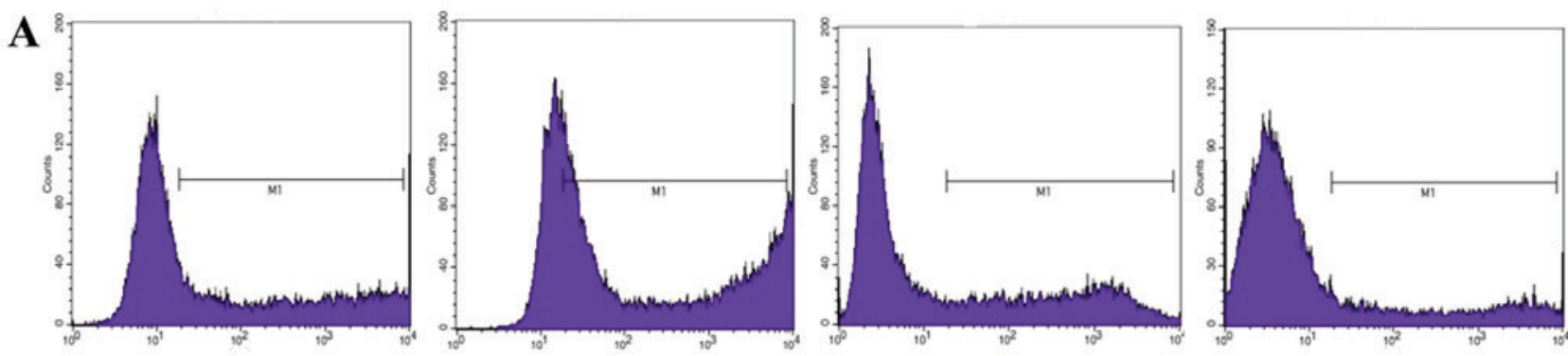

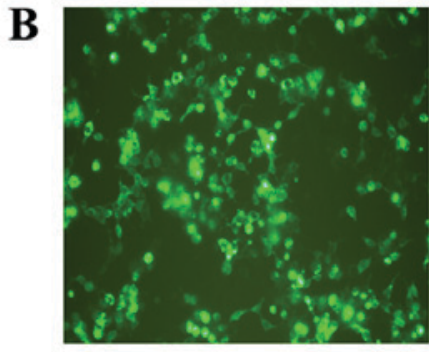

UTMD+pDNA

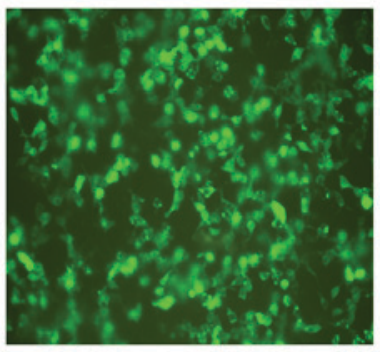

UTMD+pDNA+cNLS

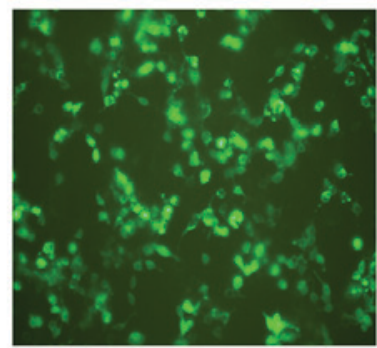

UTMD+pDNA+mNLS

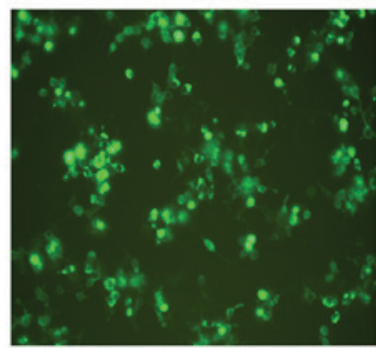

UTMD+pDNA+cNLS+WGA

Figure 7. Evaluation of transfection efficiency by (A) flow cytometry and (B) fluorescence microscopy in control group (UTMD + pDNA), cNLS group $(\mathrm{UTMD}+$ pDNA + cNLS), mNLS group (UTMD + pDNA + mNLS), and WGA (UTMD + pDNA + cNLS + WGA) group. Positive fluorescence signal is evident in the M1 portion of the flow cytometry plots, and by green signal in the microscopy images (magnification, x100). UTMD, ultrasound-targeted microbubble destruction; pDNA, plasmid DNA; NLS, nuclear localization signal; c, classic; m, mutated; WGA, wheat germ agglutinin.

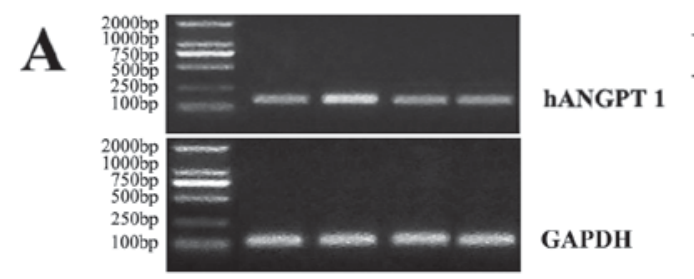

B
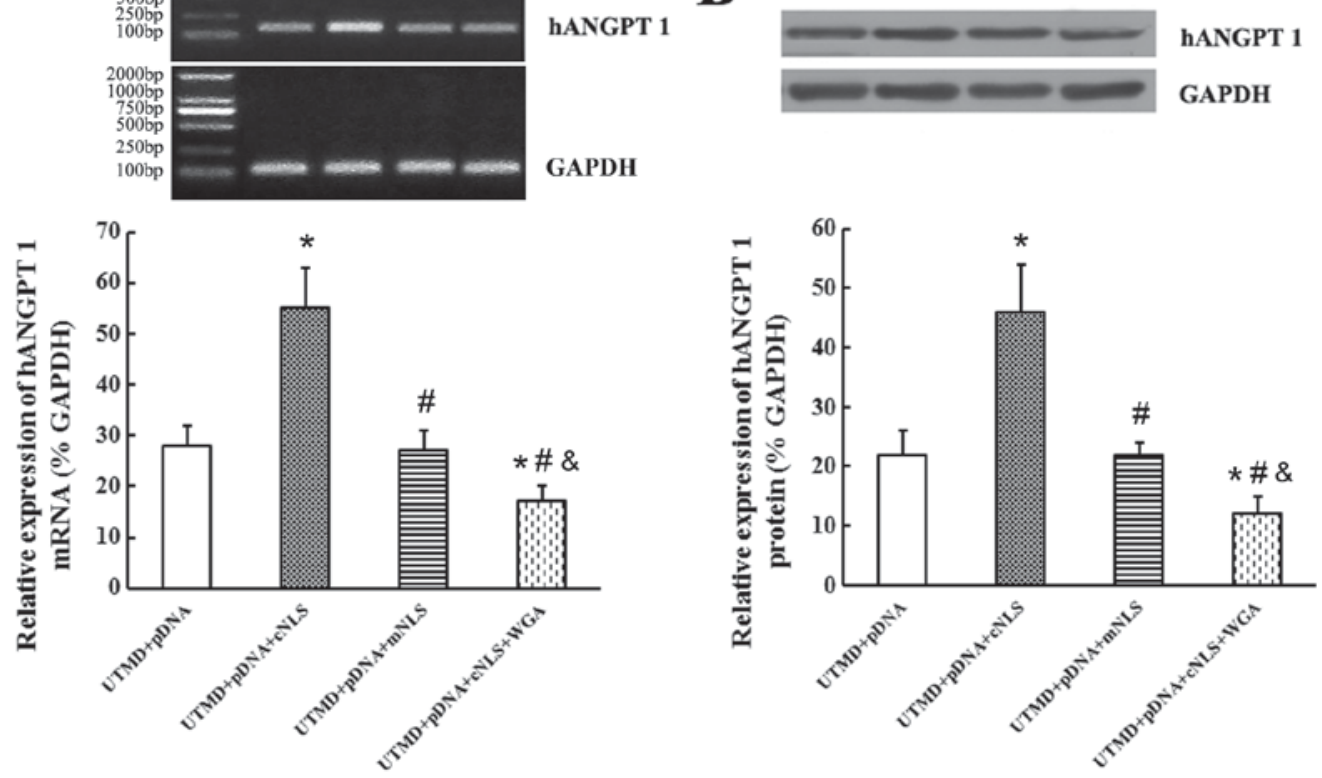

Figure 8. Evaluation of transfection efficiency by measuring expression of the target gene hANGPT1. (A) Relative expression of mRNA was measured by reverse-transcription polymerase chain reaction at $48 \mathrm{~h}$ post-transfection in the four experimental groups. (B) Relative expression of protein hANGPT1 was measured by western blotting. ${ }^{*} \mathrm{P}<0.05$ vs. control; ${ }^{*} \mathrm{P}<0.05$ vs. cNLS; and ${ }^{\&} \mathrm{P}<0.05$ vs. mNLS. hANGPT1, human angiopoietin 1 ; UTMD, ultrasound-targeted microbubble destruction; pDNA, plasmid DNA; NLS, nuclear localization signal; c, classic; m, mutated; WGA, wheat germ agglutinin.

the target gene. The present finding indicated that NLS may be important in transfection efficiency by UTMD method.

Previously, methods of exogenous gene transfection have had great progress using the function of NLS peptides $(28,29)$. For example, in nerve cells that are difficult to transfect, previous studies have performed fusion expression of an NLS peptide with a target gene, and reported that recombinant transcription factors were efficiently transferred into the neural stem cells and successfully induced nerve cell differentiation (30). In addition to vertebrates, NLS peptides can improve the transfection efficiency in crustaceans; compared with DNA alone, the non-covalent bound DNA/NLS group exhibited $>2$-fold greater transfection efficiency, faster nuclear input rate as detected by $\beta$-galactose activity determination (the progress is $20 \mathrm{~min}$ vs. 90 min in the control group), and decreased degradation by DNA hydrolytic enzymes (31). The use of cNLS in the present 
study may have enhanced the transfection efficiency by to promoting the entry of the exogenous DNA into the nucleus in great quantities, as well as avoiding exogenous DNA degradation in the cytoplasm. Thus, the combination of the two kinds of effects may significantly improve transfection efficiency.

However, various studies have adopted different experimental methods; the transfection efficiency was not always improved by combining an NLS peptide with the target gene. As such, the characteristics and advantages of the NLS peptide need be thoroughly investigated in order to explore how to optimally use NLS as a nuclear guide to enhance the rate of exogenous DNA entry into the nucleus and to thus improve the transfection efficiency in the future.

A limitation of the present study was the method of nuclei staining. Although the nuclei are displayed under a fluorescent microscope, the location of the cell nucleus is visibly more apparent if cells are co-stained with DAPI. This methodology will be further improved in the group's future investigations. Nevertheless, the present study demonstrated that the UTMD method increased cell membrane permeability for exogenous DNA entry into the cytoplasm, and addition of NLS promoted pDNA entry into the nucleus, thereby improving the overall transfection efficiency. The findings suggest that NLS combination with UTMD method may be a novel efficient strategy of non-viral vector gene transfection.

\section{Acknowledgements}

The present study was supported by the National Natural Science Foundation of China (grant nos. 81471674 and 81501495) and the Fundamental Research Funds for the Central Universities (grant no. 2015301020203).

\section{References}

1. Noble ML, Kuhr CS, Graves SS, Loeb KR, Sun SS, Keilman GW, Morrison KP, Paun M, Storb RF and Miao CH: Ultrasound-targeted microbubble destruction-mediated gene delivery into canine livers. Mol Ther 21: 1687-1694, 2013.

2. Hernot $S$ and Klibanov AL: Microbubbles in ultrasound-triggered drug and gene delivery. Adv Drug Deliv Rev 60: 1153-1166, 2008.

3. Fan Z, Chen D and Deng CX: Improving ultrasound gene transfection efficiency by controlling ultrasound excitation of microbubbles. J Control Release 170: 401-413, 2013.

4. Teulon JM, Delcuze Y, Odorico M, Chen SW, Parot P and Pellequer JL: Single and multiple bonds in (strept)avidin-biotin interactions. J Mol Recognit 24: 490-502, 2011.

5. Kocbek P, Obermajer N, Cegnar M, Kos J and Kristl J: Targeting cancer cells using PLGA nanoparticles surface modified with monoclonal antibody. J Control Release 120: 18-26, 2007.

6. Laing ST and McPherson DD: Cardiovascular therapeutic uses of targeted ultrasound contrast agents. Cardiovasc Res 83: 626-635, 2009.

7. Suzuki R, Oda Y, Utoguchi N and Maruyama K: Progress in the development of ultrasound-mediated gene delivery systems utilizing nano- and microbubbles. J Control Release 149: 36-41, 2011.

8. Mannell H, Pircher J, Fochler F, Stampnik Y, Räthel T, Gleich B, Plank C, Mykhaylyk O, Dahmani C, Wörnle M, et al: Site directed vascular gene delivery in vivo by ultrasonic destruction of magnetic nanoparticle coated microbubbles. Nanomedicine 8: 1309-1318, 2012.

9. Meinema AC, Poolman B and Veenhoff LM: The transport of integral membrane proteins across the nuclear pore complex. Nucleus 3: 322-329, 2012

10. Marfori M, Mynott A, Ellis JJ, Mehdi AM, Saunders NFW, Curmi PM, Forwood JK, Bodén M and Kobe B: Molecular basis for specificity of nuclear import and prediction of nuclear localization. Biochim Biophys Acta 1813: 1562-1577, 2011.
11. Lupberger J, Schaedler S, Peiran A and Hildt E: Identification and characterization of a novel bipartite nuclear localization signal in the hepatitis B virus polymerase. World J Gastroenterol 19: 8000- 8010, 2013.

12. Adam SA, Lobl TJ, Mitchell MA and Gerace L: Identification of specific binding proteins for a nuclear location sequence. Nature 337: 276-279, 1989.

13. Collins E, Birchall JC, Williams JL and Gumbleton M: Nuclear localisation and pDNA condensation in non-viral gene delivery. J Gene Med 9: 265-274, 2007.

14. Yoo HS and Jeong SY: Nuclear targeting of non-viral gene carriers using psoralen-nuclear localization signal (NLS) conjugates. Eur J Pharm Biopharm 66: 28-33, 2007.

15. Finlay DR, Newmeyer DD, Price TM and Forbes DJ: Inhibition of in vitro nuclear transport by a lectin that binds to nuclear pores. J Cell Biol 104: 189-200, 1987.

16. Deng P, Gong X and Jiang Y: A novel method for studying nuclear localization signal-mediated nuclear translocation. Nan Fang Yi Ke Da Xue Xue Bao 32: 1148-1150, 2012 (In Chinese).

17. Deng Q, Chen JL, Zhou Q, Hu B, Chen Q, Huang J and Guo RQ: Ultrasound microbubbles combined with the NFKB binding motif increase transfection efficiency by enhancing the cytoplasmic and nuclear import of plasmid DNA. Mol Med Rep 8: 1439-1445, 2013.

18. Villanueva FS: Getting good vibes: The therapeutic power of microbubbles and ultrasound. JACC Cardiovasc Imaging 5: 1263-1266, 2012.

19. Kooiman K, Emmer M, Foppen-Harteveld M, van Wamel A and de Jong N: Increasing the endothelial layer permeability through ultrasound-activated microbubbles. IEEE Trans Biomed Eng 57: 29-32, 2010.

20. Meijering BDM, Juffermans LJ, van Wamel A, Henning RH, Zuhorn IS, Emmer M, Versteilen AM, Paulus WJ, van Gilst WH, Kooiman K, et al: Ultrasound and microbubble targeted delivery of macromolecules is regulated by induction of endocytosis and pore formation. Circ Res 104: 679-687, 2009.

21. Bekeredjian R, Kroll RD, Fein E, Tinkov S, Coester C, Winter G, Katus HA and Kulaksiz H: Ultrasound targeted microbubble destruction increases capillary permeability in hepatomas. Ultrasound Med Biol 33: 1592-1598, 2007.

22. Li P, Gao Y, Liu Z, Tan K, Zuo Z, Xia H, Yang D, Zhang Y and Lu D: DNA transfection of bone marrow stromal cells using microbubble-mediated ultrasound and polyethylenimine: An in vitro study. Cell Biochem Biophys 66: 775-786, 2013.

23. Werth S, Urban-Klein B, Dai L, Höbel S, Grzelinski M, Bakowsky U, Czubayko F and Aigner A: A low molecular weight fraction of polyethylenimine (PEI) displays increased transfection efficiency of DNA and siRNA in fresh or lyophilized complexes. J Control Release 112: 257-270, 2006.

24. Kim BK, Kang H, Doh KO, Lee SH, Park JW, Lee SJ and Lee TJ: Homodimeric SV40 NLS peptide formed by disulfide bond as enhancer for gene delivery. Bioorg Med Chem Lett 22: 5415-5418, 2012.

25. Duvshan-Eshet M, Keren H, Oz S, Radzishevsky IS, Mor A and Machluf M: Effect of peptides bearing nuclear localization signals on therapeutic ultrasound mediated gene delivery. J Gene Med 10: 1150-1159, 2008

26. Yi WJ, Yang J, Li C, Wang HY, Liu CW, Tao L, Cheng SX, Zhuo RX and Zhang XZ: Enhanced nuclear import and transfection efficiency of TAT peptide-based gene delivery systems modified by additional nuclear localization signals. Bioconjug Chem 23: 125-134, 2012.

27. Duvshan-Eshet M, Haber T and Machluf M: Insight concerning the mechanism of therapeutic ultrasound facilitating gene delivery: Increasing cell membrane permeability or interfering with intracellular pathways? Hum Gene Ther 25: 156-164, 2014.

28. Opanasopit P, Rojanarata T, Apirakaramwong A, Ngawhirunpat T and Ruktanonchai U: Nuclear localization signal peptides enhance transfection efficiency of chitosan/DNA complexes. Int J Pharm 382: 291-295, 2009.

29. Kawazu T, Kanzaki H, Uno A, Azuma H and Nagasaki T: HVJ-E/importin- $\beta$ hybrid vector for overcoming cytoplasmic and nuclear membranes as double barrier for non-viral gene delivery. Biomed Pharmacother 66: 519-524, 2012.

30. Stock K, Nolden L, Edenhofer F, Quandel T and Brüstle O: Transcription factor-based modulation of neural stem cell differentiation using direct protein transduction. Cell Mol Life Sci 67: 2439-2449, 2010.

31. Arenal A, Pimentel R, García C, Pimentel E and Aleström P: The SV40 T antigen nuclear localization sequence enhances nuclear import of vector DNA in embryos of a crustacean (Litopenaeus schmitti). Gene 337: 71-77, 2004. 